1 Hacettepe Journal of Mathematics and Statistics

$\bigcap$ Volume 43 (6) (2014), 915-922

\title{
FG-morphisms and FG-extensions
}

\author{
Ceren Sultan ELMALI* and Tamer UGUR ${ }^{\dagger}$
}

\begin{abstract}
We investigate the relations between Fan-Gottesman compactification and categories. We deal with maps having an extension to a homeomorphism between the Fan-Gottesman compactification of their domains and ranges.
\end{abstract}

2000 AMS Classification: 54D35, 18A40.

Keywords: Fan-Gottesman compactification, categories.

Received $31: 05: 2012$ : Accepted 25 : $10: 2013$ Doi : 10.15672/HJMS.2014437526

The first section of this paper contains some preliminaries about categories. Category theory provides the language and mathematical foundations for discussing properties of large classes of mathematical objects such as the class of all sets or all groups while circumventing problems such as Russell's paradox. In fact S.Eilenberg and S. MacLane $[10,11]$ give a lot of informations about categories and functors. Category theory has also played a foundational role for formalizing new concepts such as schemes which are fundamental to major areas of contemporary research. Pioneering work of this nature was done by A.Grothendieck [7], K. Morita [12,13,14,15] and others.

The second section of this paper contains some preliminaries about the Fan-Gottesman compactification. In 1952, Ky Fan and Noel Gottesman defined a compactification that is similar to the Wallman compactification, introduced by Henry Wallman in 1938 [17], and afterwards called Fan-Gottesman compactification of regular spaces with a normal base [5]. We investigated the relations between the Fan-Gottesman and Wallman compactification and showed that Fan-Gottesman compactification of some specific and interesting spaces such as normal $A_{2}$ and $T_{4}$ is Wallman-type compactification [4]. In this section we show that Fan-Gottesman compactification can be obtained via base consisting of open ultrafilters.

*Department of Mathematics, Faculty of Science,Erzurum Technic University, Erzurum, 25240, Turkey.

Email: ceren.elmali@erzurum.edu.tr

${ }^{\dagger}$ Department of Mathematics, Faculty of Science, Ataturk University, Erzurum, 25240, Turkey.

Email:tugur@atauni.edu.tr 
In [9], Herrlich has stated that it is of interest to determine if the Wallman compactification may be regarded as a functor, especially as an epireflection functor, on a suitable category of spaces. This problem was solved affirmatively by Harris in [8].

In [3], Belaid and Echi characterize when Wallman extensions of maps are homeomorphisms.

The third section of this paper, we define $F G$-morphism and $F G$-extension. Let $X, Y$ be two $T_{3}$ spaces and $q: X \rightarrow Y$ a continuous map. An $F G$-extension of $q$ is a continuous map $F(q): F X \rightarrow F Y$ such that $F(q) \circ f_{X}=f_{Y} \circ q$, where $F X$ is the FanGottesman compactification of $X$ and $f_{X}: X \rightarrow F X$ is the canonical embedding of $X$ into its Fan-Gottesman compactification $F X$. We will characterize when Fan-Gottesman extensions of maps are homeomorphisms.

\section{Categories}

A category $C$ consist of a certain collection of object $O b(C)$ and for any two object $b, c \in O b(C)$, there is a set $\operatorname{morph}(b, c)$ of morphism (function) between $b$ and $c$. This collection may be empty, but an identity morphism $1_{b}$ must be contained in $\operatorname{morph}(b, b)$. Furthermore if there are morphism $\operatorname{morph}(b, c)$ and morphism morph $(a, b)$, then their composition must be in $\operatorname{morph}(a, c)$. Given two categories $C$ and $D$, then a map can be defined between these, the so called functor, $F: C \rightarrow D$. A functor send object of $C$ to object of $D$ and morphism in $C$ to morphism in $D$ subject to certain condition. Furthermore, it is possible to define maps between functors, the so called natural transformation [11].

One usually requires the morphisms to preserve the mathematical structure of the objects. So if the objects are all groups, a good choice for a morphism would be a group homomorphism. Similarly, for vector spaces, one would choose linear maps, and for differentiable manifolds, one would choose differentiable maps.

In the category of topological spaces, morphisms are usually continuous maps between topological spaces. However, there are also other category structures having topological spaces as objects, but they are not nearly as important as the "standard" category of topological spaces and continuous maps.

We denote by Top the category of topological spaces with continuous maps as morphisms, and by $T_{o p}$ the full subcategory of Top whose objects are the $T_{i}$ spaces. There are several ways to generalize the usual separation properties $T_{0}, T_{1}, T_{2}, T_{3}$ and $T_{4}$ of topology to topological categories [1,2]. All the above categories are full reflective subcategories of Top. There is a universal $T_{i}$-space for every topological space $X$, we denote it by $\mathbf{T}_{i}(X)$. The assignment $X \rightarrow \mathbf{T}_{i}(X)$ defines a functor $\mathbf{T}_{i}$ from Top onto Top ${ }_{i}$, which is a left adjoint functor of the inclusion functor $T_{o p} \rightarrow$ Top.

It is recalled that a continuous map $q: Y \rightarrow Z$ is said to be a quasihomeomorphism, if $U \rightarrow q^{-1}(U)$ defines a bijection $O(Z) \rightarrow O(Y)$ [7], where $O(Y)$ is the set of all open subsets of the space $Y$. If $Z$ is $T_{2}$ space and, $q$ is not onto, thus $q$ is not a quasihomeomorphism. As showed by the open sets $Z, Z \backslash\{z\}$ for some $z \in Z$. On the other hand, if $Z$ is $\mathbb{R}$, with open sets $\{(-\infty, c): c \in(-\infty, \infty]\}$ and $Y$ is its subspace $\mathbb{Q}$, then the embedding is a quasihomeomorphism. A subset $S$ of a topological space $X$ is said to be strongly dense in X, if $S$ meets every nonempty locally closed subset of $X[9]$. In here, locally closed means that every point $x$ of $S$ has a neighbourhood such that $V_{x} \cap S$ is a closed subset of $V_{x}$. In other words, $S$ is locally closed if and only if $S=O \cap F$ for some open subset $O$ of $X$ and some closed subset $F$ of $X$. In addition, one most evident definition is equivalent to closedness. Thus, a subset $S$ of $X$ is strongly dense if and only if the canonical injection $S \rightarrow X$ is a quasihomeomorphism. Besides, a continuous map 
$q: X \rightarrow Y$ is a quasihomeomorphism if and only if the topology of $X$ is the inverse image of $Y$ by $q$ and the subset $q(X)$ is strongly dense in $Y$ [7].

It is known that $T_{0}$-identification of a topological space is done by Stone [17].

Now, we will construct $T_{3}$ reflection for $X$ in Top. Firstly, we construct regular reflection by taking the supremum of all regular topologies which are coarser than the topology of $X$. This is a bireflection in Top, in other words, the underlying set stays the same. Then, apply it to the $T_{0}-$ reflection. We get a space which is regular and $T_{0}$, hence regular and $T_{1}$. The composite of the two reflection is $T_{3}$-reflection.

Let $X$ be a topological space and define $\sim$ on $X$ by $x \sim y$ if and only if $c_{X}\{x\}=$ $c l_{X}\{y\}$. Then, $\sim$ is an equivalence relation on $X$ and the resulting quotient space $X / \sim$ is $T_{0}$-space. This procedure and the space it produces are referred to as the $T_{0}$-identification of $X$. Clearly $\mathbf{T}_{0}(X)=X / \sim$. $\mathbf{T}_{0}(X)$ is called $T_{0}-$ reflection. The canonical onto map from $X$ onto its $T_{0}$ - identification $\mathbf{T}_{0}(X)$ will be denoted by $\mu_{X}$. It is clear that $\mu_{X}$ is an onto quasihomeomorphism. If $q: X \rightarrow Y$ is a continuous map,

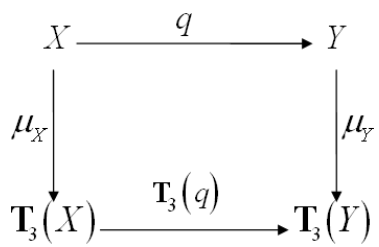

then the diagram is commutative. $\mathbf{T}_{0}$ defines a (covariant) functor from Top to itself. Thus, we get a space which is regular and $T_{0}$, hence regular and $T_{1}$. The composite of the two reflections is $T_{3}$-reflection.

\section{Fan-Gottesman Compactification}

A compactification of a topological space $X$ is a compact Hausdorff space $Y$ containing $X$ as a subspace such that $c l_{Y} X=Y$. In addition there are a lot of compactification methods applying different topological space such as Aleksandrov (one-point), Wallman, Stone-Cech. But, we study with Fan-Gottesman compactification.

Let $\beta$ be a class of open sets in $X$. If it satisfies the following three conditions, it is called a normal base.

(1) $\beta$ is closed under finite intersections

(2) If $B \in \beta$, then $X-c l_{X} B \in \beta$, where $c l_{X} B$ denotes the closure of $B$ in $X$.

(3) For every open set $U$ in $X$ and every $B \in \beta$ such that $c l_{X} B \subset U$, there exists a set $D \in \beta$ such that $c l_{X} B \subset D \subset c l_{X} D \subset U$.

We consider a regular space having a normal base for open sets i.e., which satisfies the above three properties of normal base. A chain family on $\beta$ is a non-empty family of sets of $\beta$ such that

$$
c l_{X} B_{1} \cap c l_{X} B_{2} \cap \ldots \cap c l_{X} B_{n} \neq \varnothing
$$

for any finite number of sets $B_{i}$ of the family. Every chain family on $\beta$ is contained in at least one maximal chain family on $\beta$ by Zorn's lemma. Maximal chain families on $\beta$ will be denoted by letters as $a^{*}, b^{*}, \ldots$, and also the set of all maximal chain families on $\beta$ will be denoted by $(X, \beta)^{*}$. Whose topology is defined as follow. For each $B \in \beta$, let

$$
\tau(B)=\left\{b^{*} \in(X, \beta)^{*}: \text { there exists a } A \in b^{*} \text { with } c_{X} B \subset A\right\}
$$


Then, the topology of $(X, \beta)^{*}$ is defined by taking

$$
\beta^{*}=\{\tau(B): B \in \beta\}
$$

as a base of open sets. $(X, \beta)^{*}$ is a compact Hausdorff space and is a compactification of our regular space. Afterwards this compactification is called Fan-Gottesman compactification [6].

Now, we determine the Fan-Gottesman compactification via open ultrafilters.

2.1. Definition. Let $X$ be a $T_{3}$ space and $F X$ the subcollection of all maximal ultrafilter of closed subsets on $X$. For each open set $O \subset X$, define $O^{*} \subset F X$ to be the set

$$
O^{*}=\left\{\hat{G} \in F X: \hat{G} \text { consists of } c l_{X} O\right\}
$$

Let $\Phi$ be the family of $O^{*}$. It is clear that $\Phi$ is the base for open sets of topology on $F X . F X$ is a compact space and it is called the Fan-Gottesman compactifications of $X$.

In order to avoid the confusion between $F X$ and $(X, \beta)^{*}$, we will use $F X$ when it regarded as Fan-Gottesman compactification of $X$.

On the other hand, for each closed set $D \subset X$, we define $D^{*} \subset F X$ by $D^{*}=$ $\{\hat{G} \in F X: \hat{G}$ consists of $G \subseteq D$ for some $G\}$. The following properties of $F X$ are useful;

(i) If $U \subset X$ is open, then $F X-U^{*}=(F X-U)^{*}$

(ii) If $D \subset X$ is closed, then $F X-D^{*}=(F X-D)^{*}$

(iii) If $U_{1}$ and $U_{2}$ are open in $X$, then $\left(U_{1} \cap U_{2}\right)^{*}=U_{1}^{*} \cap U_{2}^{*}$ and $\left(U_{1} \cup U_{2}\right)^{*}=U_{1}^{*} \cup U_{2}^{*}$

Properties We consider the map $f_{X}: X \rightarrow F X$ defined by $f_{X}(x)=\hat{G}_{x}$, the closed ultrafilter converging to $x$ in $X$. In order to avoid the confusing between $\hat{G}_{x}$ and $\hat{G}$, we will use $\hat{G}_{x}$ when it regarded as the maximal filter of closures of open sets containing $x$. Then the following properties hold.

(1) If $U$ is open in $X$, then $\overline{f_{X}(U)}=U^{*}$. In particular $f_{X}(X)$ is dense in $F X$.

(2) $f_{X}$ is continuous and it is an embedding of $X$ in $F X$ if and only if $X$ is a $T_{3}$-space.

(3) If $U_{1}$ and $U_{2}$ are open subsets of $X$, then $\overline{f_{X}\left(U_{1} \cap U_{2}\right)}=\overline{f_{X}\left(U_{1}\right)} \cap \overline{f_{X}\left(U_{2}\right)}$.

(4) $F X$ is a compact $T_{2}$-space .

For a $T_{3}$ space, we define $F G X=F(X)$ and we call it the Fan-Gottesman compactification of $X$. The notation $F X$ is reserved only for $T_{3}$ spaces so that it is better to use some other notation for topological spaces. The same for $f_{X}: f_{X}$ is reserved for topological space; for $\mathbf{T}_{3}$ space, we define $F_{X}=f_{X} \circ \mu_{x}$ where $\mu_{x}$ is the canonical onto map from $X$ onto its $T_{3}-$ reflection, $\mathbf{T}_{3}(X)$.

Since $\mu_{x}$ is an onto quasihomeomorphism, one obtains immediately that $F G X$ can be described exactly as $F X$ for $T_{3}$ space. The above properties are also true for a $\mathbf{T}_{3}$ space.

2.2. Remark. Let $X$ be a $T_{3}$ space. Then, the following properties hold:

(1) For each open subset $U$ of $X$, we have $F_{X}(U) \subseteq U^{*}$

(2) For each closed subset $C$ of $X$, we have $F_{X}(C) \subseteq C^{*}$

(3) Let $U$ be open and $C$ closed in a $T_{3}$ space. Then, $U \cap C \neq \varnothing$ if and only if $U^{*} \cap C^{*} \neq \varnothing$

2.3. Proposition. Let $X$ be a $T_{3}$ space and

(1) $U$ be an open subset of $X$. If $U$ is compact, then $U^{*}=F_{X}(U)$.

(2) $V$ be a closed subset of $X$. If $V$ is compact, then $V^{*}=F_{X}(V)$. 
Proof. Suppose that $V$ is closed in $X$. We have $F_{X}(V) \subseteq V^{*}$ from Remark 1. If $\hat{G} \in V^{*}$, then there exists $G \in \hat{G}$ such that $G \subseteq V$. Then $V-G$ is compact by compactness of $V$. Thus $\cap\{H \cap(V-G): H \in \hat{G}\} \neq \varnothing$. If $x \in \cap\{H \cap(V-G): H \in \hat{G}\}$, then $\hat{G}=$ $F_{X}(x)$. Hence, $\hat{G} \in F_{X}(V)$. Thus, $V^{*} \subseteq F_{X}(V)$. Therefore, $V^{*}=F_{X}(V)$.

Now, suppose that $U$ is open in $X$. Let $\hat{G} \in U^{*}$. Thus, $U \in \hat{G}$. Since, $\cap\{H: H \in \hat{G}\} \neq$ $\varnothing$, we take an $x \in \cap\{H: H \in \hat{G}\}$. It is seen that $\hat{G}=F_{X}(x)$. Therefore, according to Remark $1, U^{*}=F_{X}(U)$.

\section{FG-morphisms and $F G$-extensions}

Recall from [3] that a subset $S$ of a topological space $X$ is said to be sufficiently dense if $S$ meets each nonempty closed subset and each nonempty open subset of $X$. By an almost -homeomorphism ( $\alpha$-homeomorphism, for short), we mean a continuous map $q: X \rightarrow Y$ such that $q(X)$ is sufficiently dense in $Y$ and the topology of $X$ is the inverse image of $Y$ by $q$.

3.1. Definition. i) A subset $C$ of a topological space is said to be openly dense if $C$ meets each nonemty open subset of $X$.

Thus we have the following implications:

$$
\begin{gathered}
\text { Strongly dense } \Rightarrow \text { Sufficiently dense } \Rightarrow \text { openly dense } \\
\Downarrow \\
\text { Dense }
\end{gathered}
$$

3.2. Definition. By a Fan-Gottesman morphism ( $F G$-morphism, for short), we mean a continuous map $q: X \rightarrow Y$ such that $q(X)$ is openly dense in $Y$ and the topology of $X$ is the inverse image of $Y$ by $q$. We conclude that

homeomorphism $\Rightarrow$ quasihomeomorphism $\Rightarrow \alpha$-homeomorphism $\Rightarrow F G$-morphism

\subsection{Theorem.}

(1) The composition of two FG-morphisms is an FG-morphism.

(2) If $q: X \rightarrow Y$ is an FG-morphism and $X$ is $T_{0}$, then $q$ is injective.

(3) If $q: X \rightarrow Y$ is an FG-morphism and $Y$ is $T_{1}$, then $q$ is an onto homeomorphism.

(4) If $q: X \rightarrow Y$ is an FG-morphism, $X$ is $T_{0}$ and $Y$ is $T_{1}, q$ is a homeomorphism.

Proof. We show that (1). Let $p: X \rightarrow Y$ and $q: Y \rightarrow Z$ be two $F G$-morphisms. Clearly, the topology of $X$ is the inverse image of $Z$ by $q \circ p$. Let $A$ be open subset of $Z$. Since $q^{-1}(A)$ is open in $Y$, the $p(x) \cap q^{-1}(A) \neq \varnothing$, so that $A \cap q(p(X)) \neq \varnothing$. Hence, $q \circ p$ is an $F G$-morphism.

(2) Let $x_{1}, x_{2}$ be two points of $X$ with $q\left(x_{1}\right)=q\left(x_{2}\right)$. Suppose that $x_{1} \neq x_{2}$. Then, there exists an open subset $U$ of $X$ such that $x_{1} \in U, x_{2} \notin U$, since $X$ is $T_{0}$. Because there exists an open subset $H$ of $Y$ satisfying $q^{-1}(H)=U$, we get $q\left(x_{1}\right) \in H$ and $q\left(x_{2}\right) \notin H$, which is impossible. It follows that $q$ is injective.

(3) Let $y \in Y$. Then, $\{y\}$ is a locally closed subset of $Y$. Hence, $\{y\} \cap q(X) \neq \varnothing$, since $q(X)$ is strongly dense in $Y$. Thus, $y \in q(X)$, hence $q$ is an onto map.

(4) It is clear that $q$ is homeomorphism from (2) and (3).

Now, we define $F G$-extensions.

3.4. Definition. A continuous map $q: X \rightarrow Y$ between $T_{3}$ spaces is said to be an $F G$ extension, if there is a continuous map $F(q): F X \rightarrow F Y$ such that $f_{Y} \circ q=F(q) \circ f_{X}$. 
3.5. Theorem. Let $X, Y$ be two $T_{3}$ spaces and $q: X \rightarrow Y$ an FG-morphism. Then, $q$ has an FG-extension which is a homeomorphism.

Proof. We remark that diagram in the introduction commutes. Hence, $\mathbf{T}_{3}(q) \circ \mu_{x}=\mu_{y} \circ q$. Thus, $\mathbf{T}_{3}(q) \circ \mu_{x}$ is an $F G$-morphism. Now, $\mathbf{T}_{3}(q)$ is an $F G$-morphism from Proposition 2.1, since $\mu_{x}$ is a quasihomeomorphism. Therefore, $\mathbf{T}_{3}(q)$ is a homeomorphism by Proposition 2.1. It follows that $\mathbf{T}_{3}(q)$ has a canonical $F G$-extension $F\left(\mathbf{T}_{3}(q)\right)$ which is a homeomorphism. Thus, the diagram commutes. If we denote $F G(q)=F\left(\mathbf{T}_{3}(q)\right)$, then the diagram indicates clearly that $F G(q)$ is an $F G$-extension of $q$ which is a homeomorphism.

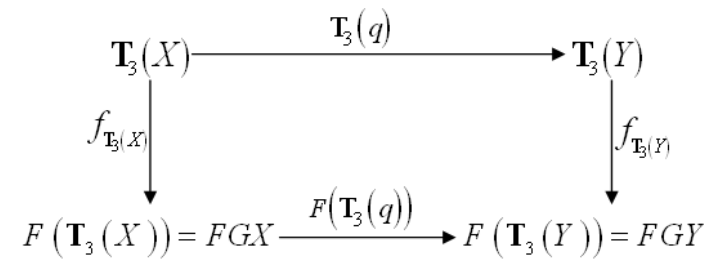

It is known that if $X$ is a $T_{4}$ space, then $F G X=w X=\beta(X)$ (the Wallman and Stone-Čech compactification, respectively)[4].

3.6. Corollary. If $\mathbf{T}_{3}(X)$ is a $T_{4}$ space, then $F G X=w\left(\mathbf{T}_{3}(q)\right)=\beta\left(\mathbf{T}_{3}(q)\right)$.

3.7. Definition. Let $X$ be a $T_{3}$ space and $Y$ a subspace of $X$.

(1) $Y$ is called a Fan-Gottesman generator ( $F G$-generator) of $X$, if $F G Y$ is homeomorphic to $F G X$.

(2) $Y$ is called a strong Fan-Gottesman generator ( $s F G$-generator) of $X$, if the canonical embedding $i: Y \rightarrow X$ has an $F G$-extension $F G(i)$ which is a homeomorphism.

Clearly, $s F G$-generator $\Rightarrow F G$-generator

3.8. Theorem. Let $X, Y$ be two $T_{3}$ spaces and $q: X \rightarrow Y$ a continuous map. Then, the following statements are equivalent:

(1) $q$ has an $F G$-extension which is a homeomorphism.

(2) $q(X)$ is an $s F G$-generator of $Y$ and the topology of $X$ is the inverse image of $Y$ by $q$.

Proof. $\quad(i) \Rightarrow(i i)$ Firstly, we show that the topology of $X$ is the inverse image of $Y$ by $q$. Let $U$ be an open subset of $X$. Since $F G(q)$ is a homeomorphism, $F G(q)\left(U^{*}\right)=V$ is a closed subset of $w Y$. Set $G=F_{y}^{-1}(V)$. We prove that $U=q^{-1}(G)$.

(a) Let $x \in U$. Then, $F_{X}(x) \in F_{X}(U) \subseteq U^{*}$. Hence, $F G(q)\left(F_{X}(x)\right) \in F G(q)\left(U^{*}\right)=$ $V$ which gives $F_{Y}(q(x)) \in V$. It follows that $q(x) \in F_{Y}^{-1}(V)=G$. Therefore, $x \in$ $q^{-1}(G)$.

(b) Conversely, let $x \in q^{-1}(G)$. Then, $q(x) \in G=F_{X}^{-1}(V)$; this means that $\left(F_{Y} \circ q\right)(x) \in V$, so that $F G(q)\left(F_{X}(x)\right) \in V=F G(q)\left(U^{*}\right)$. Since $F G(q)$ is bijective, $F_{X}(x) \in U^{*}$. Hence, $x \in F_{X}^{-1}\left(U^{*}\right)=U$. We have proved that $U=q^{-1}(G)$. In other words, the topology of $X$ is the inverse image of $Y$ by $q$.

Secondly, we show that $q(X)$ is an $s F G$-generator of $Y$. According to (1), the map $q_{1}: X \rightarrow q(X)$ induced by $q$ is an $F G$-morphism. Hence, $q_{1}$ has an $F G$-extension $F\left(q_{1}\right)$ which is a homeomorphism, by Proposition 2.1. Thus, the diagrams commute. 


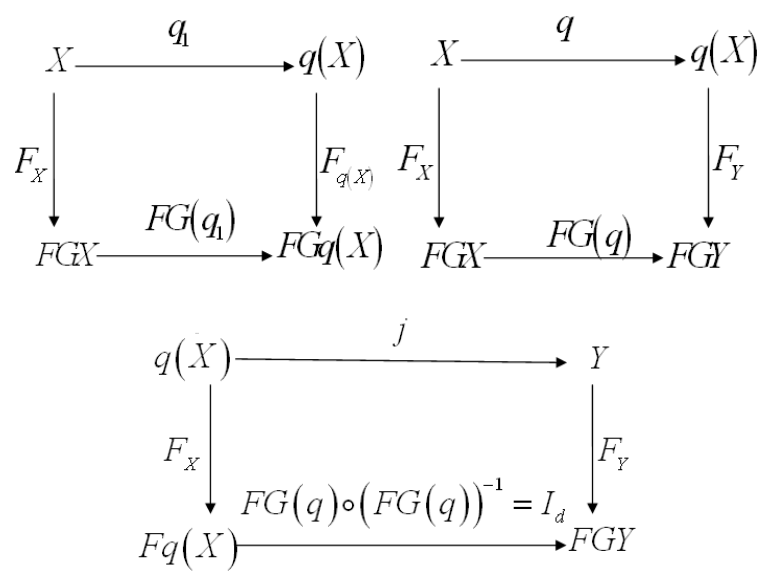

Let $j: q(X) \rightarrow Y$ be the canonical embedding. Clearly, the diagram commutes.

Therefore, $j$ has $F G(q) \circ(F G(q))^{-1}=I_{d}$ as an $F G$-extension which is a homeomorphism. This means that $q(X)$ is an $s F G$ generator of $Y$. (ii $) \Rightarrow(i)$ We assume $(i i)$. The map $q_{1}: X \rightarrow q(X)$ induced by $q$ is an $F G$-morphism. Thus, according to Proposition 2.1, $q_{1}$ has an $F G$-extension $F\left(q_{1}\right)$ which is a homeomorphism. On the other hand, the canonical embedding $j: q(X) \rightarrow Y$ has an $F G$-extension which is a homeomorphism, by Proposition 2.1. It follows that the two diagrams commute.

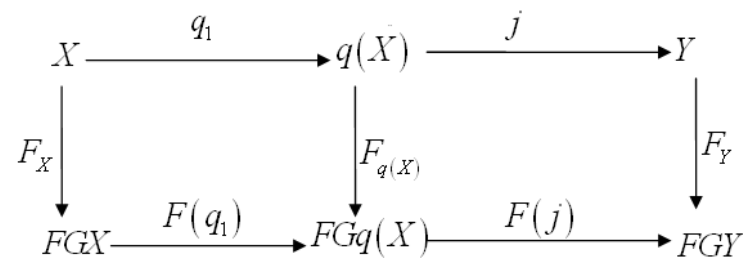

Therefore, $F(j) \circ F\left(q_{1}\right)$ is an $F G$-extension of $q: X \rightarrow Y$ which is a homeomorphism.

Theorem 3.4 seems us to the following classical fact about the Stone-Cech compactification $e_{X}: X \rightarrow \beta X$ of a Tychonoff space $X$.

Consider any continuous mapping $p: X \rightarrow Y$, where $Y$ is also Tychonoff. Then, the map $\beta(p): \beta X \rightarrow \beta Y$ is a homeomorphism if and only if $p$ is a dense $C^{*}$-embedding.

We can mention this analogy in our paper.

3.9. Theorem. If $X$ and $Y$ are Tychonoff spaces, then the following are equivalent for a map $f: X \rightarrow Y$;

(1) $F(f)[F X \backslash X]$ is contained in $F Y \backslash Y$.

(2) The diagram

is pullback.

Proof. (1) $\Rightarrow(2)$ Suppose that $h: Z \rightarrow F X$ and $g: Z \rightarrow Y$ are mapping such that $F(f) \circ h=f_{Y} \circ g$. Since $f_{Y} \circ g[Z]$ is contained in $F Y$ and $F(f)$ sends $F X \backslash X$ into $F Y \backslash Y$, we have that $h[Z]$ is contained in $X$. Hence, defining $I: Z \rightarrow X$ by $I(z)=h(z)$, 


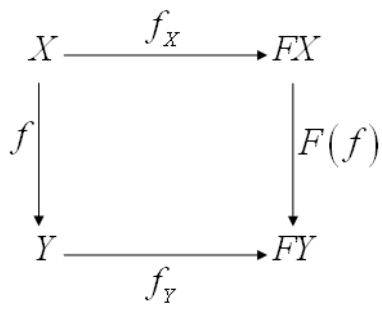

it is shown that the square is pullback.(2) $\Rightarrow(1)$ Choose $p$ in $F X$ and assume that $F(f)(p)=y$ belongs to $Y$. Then, let $h$ be the map which embeds $\{p\}$ into $F X$ and $g$ be the map from the subspace $\{p\}$ which sends $p$ to $F(f)(p)$. Then, $F(f) \circ h=f_{Y} \circ g$ so that there exist a map $I:\{p\} \rightarrow X$ such that $h=f_{x} \circ I$. Hence, $p$ belongs to $X$.

\section{References}

[1] M. Baran, Separation Properties, Indian J. Pure and Appl. Math. 23, 333-342 (1992).

[2] M. Baran and H. Altındiş, $T_{2}$-objects in topological categories, Acta Math. Hungarica 71, 41-48 (1996).

[3] K. Belaid and O. Echi, $T_{(\alpha, \beta)}$-Space and the Wallman Compactification, International Journal of Mathematics and Mathematical Sciences Vol.2004, Issue 68, 3717-3735 (2004).

[4] C.Elmalı and T.Uğur, Fan-Gottesman Compactification of some specific space is Wallmantype compactification, Chaos,Soliton and Fractals, Vol.42, no.1, 17-19 (2009).

[5] K. Fan and N. Gottesman, On compactifications of Freudenthal and Wallman, Indag. Math. 14, 504-510 (1952).

[6] G. Gierz, K. H. Hofmann, K. Keimel, J. D. Lawson, M. W. Mislove and D. S. Scott, Continuous Lattices and Domains, Cambridge University Press, Cambridge, England (2003).

[7] A. Grothendieck and J. Dieudonné, Éléments de Géometrie Algébrique, Die Grundlehren der mathematischen Wissenchaften, vol.166, Springer-Verlag, New York, (1971).

[8] D. Harris, The Wallman compactification is an epireflection, Proc. Amer. Math. Soc. 31, 265-267 (1972).

[9] H. Herrlich, On the concept of reflections in general topology, Contributions to Extension Theory of Topological Structures (Proc. Sympos., Berlin, 1967), Deutsch. Verlag Wissensch., Berlin, 105-114 (1969).

[10] S. Maclane, Categories for the Working Mathematician, Springer-Verlag, New York, (1971).

[11] S.Maclane and S.Eilenberg, Eilenberg-Maclane:collected works, Academic Press Inc., Orlando, FL, (1986).

[12] K.Morita, Category-isomorphism and endomorphism rings of modules, Trans. Amer. Math. Soc. 103, 451-469 (1962).

[13] K.Morita, Localization in categories of modules I. Math. Z. 114, 121-144 (1970).

[14] K.Morita, Localization in categories of modules II. J. Reine Angew. Math. 242, 163-169 (1970).

[15] K.Morita, Localization in categories of modules III. Math. Z. 119, 313-320 (1971).

[16] M. H. Stone, Applications of Boolean algebras to general topology, Bull. Amer. Math. Soc. 41, 375-481 (1997).

[17] H. Wallman, Lattices and topological spaces, Ann. of Math. (2) 39, 112-126 (1938). 